\title{
Theoretical Study on Fluid Velocity for Viscous Fluid in a Circular Cylindrical Shell
}

\author{
Hao Yajuan*, Shi Yunhui and Ping Panpan \\ School of Science, Yanshan University, Qinhuangdao, China
}

\begin{abstract}
A theoretical algorithm by united Lagrangian-Eulerian method for the problem in dealing with viscous fluid and a circular cylindrical shell is presented. In this approach, each material is described in its preferred reference frame. Fluid flows are given in Eulerian coordinates whereas the elastic circular cylindrical shell is treated in a Lagrangian framework. The fluid velocity in a two-dimensional uniform elastic circular cylindrical shell filled with viscous fluid is studied under the assumption of low Reynolds number. The coupling between the viscous fluid and the elastic circular cylindrical shell shows kinematic conditions at the shell surface. Also, the radial velocity and axial velocity of the fluid are discussed with the help of graphs.
\end{abstract}

Keywords: Elastic circular cylindrical shell, fluid velocity, kinematic condition, united lagrangian-eulerian method, viscous fluid.

\section{INTRODUCTION}

This paper deals with the mathematical analysis of problem for viscous fluid and a circular cylindrical shell by a new theoretical method. These phenomena are of major importance for aerospace, ocean engineering, mechanical or biomedical applications, etc. and thus have been studied by many authors over the past few years [1-4]. The situation has mainly been analysed by numerical methods $[5,6]$. Our focus is the velocity of the fluid when the elastic circular cylindrical shell filled with viscous fluid vibrates.

Typically, the viscous fluid and a circular cylindrical shell are given in different coordinate systems making a common solution. Fluid flows are given in Eulerian coordinates whereas the circular cylindrical shell is treated in a Lagrangian framework. United Lagrangian-Eulerian method is used to present the flow velocity of a viscous and incompressible fluid in a circular cylindrical shell. It is a new method of where fluid and circular cylindrical shell equations are given in their preferred reference frames. Coupling between the fluid and the circular cylindrical shell domains represents kinematic conditions at the shell surface. The two-dimensional problem is that of an elastic circular cylindrical shell in which waves of lateral displacement are propagated.

\section{BASIC EQUATIONS FOR VISCOUS FLUID}

In this study, the analysis of the fluid velocity of an incompressible viscous fluid in a complete circular cylindrical shell of radius $R$ is conducted. The shell is horizontal. In order to describe the geometry of the shell, a

*Address correspondence to this author at the School of Science, Yanshan University, Qinhuangdao, China; Tel: +86-13582903299;

E-mail: Yajuan13582903299@qq.com cylindrical coordinates system $(O ; r, \theta, z)$ is considered, where $O$ is the origin placed at the centre of the shell, $r$ is the radial and $z$ is the axial coordinate. The displacement field of the middle surface of the shell is given by the following components: $u_{r}, u_{\theta}$ and $u_{z}$; in the radial, circumferential and axial directions, respectively.

In cylindrical coordinates system, the Navier-Stokes equations of incompressible viscous fluid can be expressed as

$$
\left\{\begin{array}{l}
\rho\left(\frac{d v_{r}}{d t}-\frac{v_{\theta}^{2}}{r}\right)=\rho F_{r}-\frac{\partial p}{\partial r}+\mu\left(\nabla^{2} v_{r}-\frac{v_{r}}{r^{2}}-\frac{2}{r^{2}} \frac{\partial v_{\theta}}{\partial \theta}\right) \\
\rho\left(\frac{d v_{\theta}}{d t}+\frac{v_{r} v_{\theta}}{r}\right)=\rho F_{\theta}-\frac{\partial p}{r \partial \theta}+\mu\left(\nabla^{2} v_{\theta}-\frac{v_{\theta}}{r^{2}}+\frac{2}{r^{2}} \frac{\partial v_{r}}{\partial \theta}\right) \\
\rho \frac{d v_{z}}{d t}=\rho F_{z}-\frac{\partial p}{\partial z}+\mu \nabla^{2} v_{z}
\end{array}\right.
$$

where $\mu$ is the coefficient of viscosity, $\rho$ is the mass density of the fluid, $p$ is the pressure, $v_{r}, v_{\theta}$ and $v_{z}$ are the projections of the velocity vector, $F_{r}, F_{\theta}$ and $F_{z}$ are the projections of the unit mass force vector, $t$ is time. In equations (1), the operators are defined as

$$
\begin{aligned}
& \frac{d}{d t}=\frac{\partial}{\partial t}+v_{r} \frac{\partial}{\partial r}+v_{\theta} \frac{\partial}{\partial \theta}+v_{z} \frac{\partial}{\partial z} \\
& \nabla^{2}=\frac{1}{r} \frac{\partial}{\partial r}\left(r \frac{\partial}{\partial r}\right)+\frac{1}{r^{2}} \frac{\partial^{2}}{\partial \theta^{2}}+\frac{\partial^{2}}{\partial z^{2}}
\end{aligned}
$$

The Navier-Stokes equations of incompressible viscous fluid are almost equal to the Stokes equations under the assumption of low Reynolds number. The Stokes equations can be written as 


$$
\left\{\begin{array}{l}
\frac{\partial p}{\partial r}=\mu\left(\nabla^{2} v_{r}-\frac{v_{r}}{r^{2}}-\frac{2}{r^{2}} \frac{\partial v_{\theta}}{\partial \theta}\right) \\
\frac{\partial p}{r \partial \theta}=\mu\left(\nabla^{2} v_{\theta}-\frac{v_{\theta}}{r^{2}}+\frac{2}{r^{2}} \frac{\partial v_{r}}{\partial \theta}\right) \\
\frac{\partial p}{\partial z}=\mu \nabla^{2} v_{z}
\end{array}\right.
$$

The continuity equation for the viscous fluid can be described as

$$
\frac{1}{r} \frac{\partial\left(r v_{r}\right)}{\partial r}+\frac{1}{r} \frac{\partial v_{\theta}}{\partial \theta}+\frac{\partial v_{z}}{\partial z}=0
$$

\section{UNITED LAGRANGIAN-EULERIAN METHOD}

The fixed point $m$ on the shell surface coincides with the space point $M$ at $t=0$ whereas $m$ coincides with the space point $M^{*}$ at $t>0$. Adhesion condition for viscous fluid can be simplified as [7]

$$
\mathbf{V}=\frac{\partial \mathbf{u}}{\partial t}
$$

where $\boldsymbol{u}$ is the displacement vector of the fixed point $m, \mathbf{V}$ is velocity vector of space point $M^{*}$.

Dynamic condition is

$$
\mathbf{Z}=\mathbf{p}_{3}-\mathbf{p}_{3}^{\prime}
$$

where $\mathbf{p}_{3}$ and $\mathbf{p}_{3}^{\prime}$ are the pressures in different directions at the space point $M^{*}, \mathbf{Z}$ is the force vector. In the cylindrical coordinate, $V_{m}$ can be expressed as

$V_{m}=v_{m}+u_{r} \frac{\partial v_{m}}{\partial r}+u_{\theta} \frac{\partial v_{\theta}}{\mathbf{r} \partial \theta}+u_{z} \frac{\partial v_{m}}{\partial z}$

where $V_{m}(m=r, \theta, z)$ are the projections of the velocity at the space point $M^{*} \cdot v_{m}(m=r, \theta, z)$ are the projections of the velocity at the space point $M$. Taking into account the minor deformation of the shell, the third item of Taylor expansions will not be considered.

Expression (4) can be written as

$$
\frac{\partial u_{r}}{\partial t}-V_{r}=0, \frac{\partial u_{\theta}}{\partial t}-V_{\theta}=0, \frac{\partial u_{z}}{\partial t}-V_{z}=0
$$

where $V_{r}, V_{\theta}$ and $V_{z}$ can be obtained from equation (6).

\section{VELOCITY OF FLUID}

In order to simplify the analysis, only an infinite length circular cylindrical shell was considered. The waving surface of the circular cylindrical shell is is represented by:

$u_{r}=b \sin (\alpha z-\omega t), u_{z}=0$

where $\alpha=2 \pi / \lambda, \omega=c \alpha, b$ is the amplitude of the traveling wave, $\lambda$ is the wavelength, $c$ is the velocity of the wave.
Considering the Reynolds number $\operatorname{Re}<<1$, the Stokes equations in two dimensions can be written as

$\frac{\partial p}{\partial r}=\mu\left(\nabla^{2} v_{r}-\frac{v_{r}}{r^{2}}\right), \frac{\partial p}{\partial z}=\mu \nabla^{2} v_{z}$

in which

$\nabla^{2}=\frac{1}{r} \frac{\partial}{\partial r}\left(r \frac{\partial}{\partial r}\right)+\frac{\partial^{2}}{\partial z^{2}}$

The continuity equation of the incompressible viscous fluid can be described as

$\frac{1}{r} \frac{\partial\left(r v_{r}\right)}{\partial r}+\frac{\partial v_{z}}{\partial z}=0$

The stream function $\psi$ satisfies the conditions

$v_{r}=\frac{\partial \psi}{r \partial z}, v_{z}=-\frac{\partial \psi}{r \partial r}$

The kinematic conditions can be written as

$\left\{\begin{array}{l}\frac{\partial u_{r}}{\partial t}=v_{r}+u_{r} \frac{\partial v_{r}}{\partial r}+u_{\theta} \frac{\partial v_{r}}{\partial \theta}+u_{z} \frac{\partial v_{r}}{\partial z} \\ \frac{\partial u_{\theta}}{\partial t}=v_{\theta}+u_{r} \frac{\partial v_{\theta}}{\partial r}+u_{\theta} \frac{\partial v_{\theta}}{\partial \theta}+u_{z} \frac{\partial v_{\theta}}{\partial z} \\ \frac{\partial u_{z}}{\partial t}=v_{z}+u_{r} \frac{\partial v_{z}}{\partial r}+u_{\theta} \frac{\partial v_{z}}{\partial \theta}+u_{z} \frac{\partial v_{z}}{\partial z}\end{array}\right.$

Thus the following expressions for $\psi$ can be written

$\left\{\begin{array}{l}\frac{\partial \psi}{r \partial r}+u_{r} \frac{\partial}{\partial r}\left(\frac{\partial \psi}{r \partial r}\right)=0 \\ \frac{\partial \psi}{r \partial z}+u_{r} \frac{\partial}{\partial r}\left(\frac{\partial \psi}{r \partial z}\right)=\frac{\partial u_{r}}{\partial t}\end{array} \quad(r=R)\right.$

Making use of equations (9), (10) and (11), expression for stream function $\psi$ can be written as

$\left(\frac{\partial^{2}}{\partial z^{2}}+\frac{\partial^{2}}{\partial r^{2}}-\frac{1}{r} \frac{\partial}{\partial r}\right)^{2} \psi=0$

The solution of the equation (13) is

$\psi=B_{0} r^{2}+\sum_{n=1}^{N}\left[A_{n} r^{2} I_{0}(n \alpha r)+B_{n} r I_{1}(n \alpha r)\right] b \sin n(\alpha z-\omega t)$

where $I_{0}(n \alpha r), I_{1}(n \alpha r)$ are the Bessel functions of the first kind [8].

The solutions of the Bessel equation $x^{2} y^{\prime \prime}+x y^{\prime}+$ $\left(x^{2}-v^{2}\right) y=0 \quad(v \geq 0)$ is $v$ order Bessel function. A special solution of Bessel function can be written as

$$
y(x)=\sum_{k=0}^{\infty}(-1)^{k} \frac{1}{k !(k+\rho) !}\left(\frac{x}{2}\right)^{2 k+\rho} \quad(\rho=v)
$$

This is $v$ order Bessel function of the first kind. We get 


$$
\begin{aligned}
& I_{0}(x)=\sum_{k=0}^{\infty}(-1)^{k} \frac{1}{(k !)^{2}}\left(\frac{x}{2}\right)^{2 k} \\
& I_{1}(x)=\sum_{k=0}^{\infty}(-1)^{k} \frac{1}{k !(k+1) !}\left(\frac{x}{2}\right)^{2 k+1} \\
& I_{0}(n \alpha r)=\sum_{k=0}^{\infty}(-1)^{k} \frac{1}{(k !)^{2}}\left(\frac{n \alpha r}{2}\right)^{2 k} \\
& I_{1}(n \alpha r)=\sum_{k=0}^{\infty}(-1)^{k} \frac{1}{k !(k+1) !}\left(\frac{n \alpha r}{2}\right)^{2 k+1} \\
& \frac{\partial I_{0}(n \alpha r)}{\partial r}=-n \alpha I_{1}(n \alpha r), \frac{\partial\left[r I_{1}(n \alpha r)\right]}{\partial r}=n \alpha r I_{0}(n \alpha r)
\end{aligned}
$$

Substituting the equations (8) and (14) into (12), the following expressions can be written as

$$
\begin{aligned}
& 2 B_{0}+\sum_{n=1}^{N}\left[2 A_{n} I_{0}(n \alpha r)-n \alpha r A_{n} I_{1}(n \alpha r)+n \alpha B_{n} I_{0}(n \alpha r)\right] \\
& \times \sin n(\alpha z-\omega t)+b \sin (\alpha z-\omega t)\left\{2 B_{0}+\right. \\
& \sum_{n=1}^{N}\left[2 A_{n} I_{0}(n \alpha r)-3 n \alpha r A_{n} I_{1}(n \alpha r)-(n \alpha r)^{2} A_{n} I_{0}(n \alpha r)\right. \\
& \left.\left.+n \alpha B_{n} I_{0}(n \alpha r)-n^{2} \alpha^{2} r B_{n} I_{1}(n \alpha r)\right] \sin n(\alpha z-\omega t)\right\}=0 \\
& \sum_{n=1}^{N}(n \alpha)\left[A_{n} r I_{0}(n \alpha r)+B_{n} I_{1}(n \alpha r)\right] \cos n(\alpha z-\omega t)- \\
& b \sin (\alpha z-\omega t) \sum_{n=1}^{N}(n \alpha)\left[-A_{n} I_{0}(n \alpha r)+\frac{B_{n}}{r} I_{1}(n \alpha r)\right. \\
& \left.+n \alpha r A_{n} I_{1}(n \alpha r)-n \alpha B_{n} I_{0}(n \alpha r)\right] \cos n(\alpha z-\omega t)+ \\
& b \omega \cos (\alpha z-\omega t)=0
\end{aligned}
$$

Because of $z$ arbitrary, the following expressions can be written

$$
\begin{aligned}
& B_{0}=0 \\
& \sum_{n=1}^{N}\left[2 A_{n} I_{0}(n \alpha r)-n \alpha r A_{n} I_{1}(n \alpha r)+n \alpha B_{n} I_{0}(n \alpha r)\right]=0 \\
& \sum_{n=1}^{N}\left[2 A_{n} I_{0}(n \alpha r)-3 n \alpha r A_{n} I_{1}(n \alpha r)-(n \alpha r)^{2} A_{n} I_{0}(n \alpha r)\right. \\
& \left.+n \alpha B_{n} I_{0}(n \alpha r)-(n \alpha)^{2} r B_{n} I_{1}(n \alpha r)\right]=0 \\
& \sum_{n=1}^{N}(n \alpha)\left[r A_{n} I_{0}(n \alpha r)+B_{n} I_{1}(n \alpha r)\right]=0 \\
& -\alpha A_{1} I_{0}(\alpha r)+\alpha^{2} r A_{1} I_{1}(\alpha r)+\frac{\alpha B_{1}}{r} I_{1}(\alpha r)-\alpha^{2} B_{1} I_{0}(\alpha r)-2 \omega=0
\end{aligned}
$$

The following expressions for $r=R$ and $N=2$ can be written

$$
\begin{aligned}
& 2 I_{0}(\alpha R) A_{1}-\alpha R I_{1}(\alpha R) A_{1}+\alpha I_{0}(\alpha R) B_{1} \\
& +2 I_{0}(2 \alpha R) A_{2}-2 \alpha R I_{1}(2 \alpha R) A_{2}+2 \alpha I_{0}(2 \alpha R) B_{2}=0 \\
& 2 I_{0}(\alpha R) A_{1}-3 \alpha R I_{1}(\alpha R) A_{1}-(\alpha R)^{2} I_{0}(\alpha R) A_{1} \\
& +\alpha I_{0}(\alpha R) B_{1}-\alpha^{2} R I_{1}(\alpha R) B_{1}+2 I_{0}(2 \alpha R) A_{2}- \\
& 6 \alpha R I_{1}(2 \alpha R) A_{2}-(2 \alpha R)^{2} I_{0}(2 \alpha R) A_{2} \\
& +2 \alpha I_{0}(2 \alpha R) B_{2}-(2 \alpha)^{2} R I_{1}(2 \alpha R) B_{2}=0 \\
& \alpha R I_{0}(\alpha R) A_{1}+\alpha I_{1}(\alpha R) B_{1}+ \\
& 2 \alpha R I_{0}(2 \alpha R) A_{2}+2 \alpha I_{1}(2 \alpha R) B_{2}=0 \\
& -\alpha A_{1} I_{0}(\alpha R)+\alpha^{2} R A_{1} I_{1}(\alpha R)+ \\
& \frac{\alpha B_{1}}{R} I_{1}(\alpha R)-\alpha^{2} B_{1} I_{0}(\alpha R)-2 \omega=0
\end{aligned}
$$

The solutions of the set of equations (15)-(18) are $A_{1}=\frac{D_{1}}{D}, B_{1}=\frac{D_{2}}{D}, A_{2}=\frac{D_{3}}{D}, B_{2}=\frac{D_{4}}{D}$

where

$$
\begin{aligned}
& D=8\left(\alpha^{5} R^{2}-3 \alpha^{3}\right) I_{0}(\alpha R) I_{1}(\alpha R) I_{1}^{2}(2 \alpha R)+ \\
& 16 \alpha^{5} R^{2} I_{0}(\alpha R) I_{1}(\alpha R) I_{0}^{2}(2 \alpha R) \\
& -4\left(\alpha^{6} R^{3}-3 \alpha^{4} R\right) I_{1}^{2}(\alpha R) I_{1}^{2}(2 \alpha R)- \\
& \left(4 \alpha^{6} R^{3}+\alpha^{4} R\right) I_{1}^{2}(\alpha R) I_{0}^{2}(2 \alpha R)+ \\
& 8\left(\alpha^{5} R^{2}-\alpha^{3}\right) I_{0}^{2}(\alpha R) I_{0}(2 \alpha R) I_{1}(2 \alpha R)+ \\
& 4\left(\alpha^{5} R^{2}+4 \alpha^{3}\right) I_{1}^{2}(\alpha R) I_{0}(2 \alpha R) I_{1}(2 \alpha R) \\
& -8\left(\alpha^{6} R^{3}+2 \alpha^{4} R\right) I_{0}^{2}(\alpha R) I_{1}^{2}(2 \alpha R)- \\
& 4 \alpha^{6} R^{3} I_{0}^{2}(\alpha R) I_{0}^{2}(2 \alpha R)+ \\
& 8\left(\alpha^{2} / R-\alpha^{4} R\right) I_{0}(\alpha R) I_{1}(\alpha R) I_{0}(2 \alpha R) I_{1}(2 \alpha R) \\
& D_{1}=4\left(\alpha^{2} \omega-2 \alpha^{4} R^{2} \omega\right) I_{1}(\alpha R) I_{0}^{2}(2 \alpha R)- \\
& 16 \alpha^{3} R \omega I_{1}(\alpha R) I_{0}(2 \alpha R) I_{1}(2 \alpha R) \\
& -8 \alpha^{4} R^{2} \omega I_{1}(\alpha R) I_{1}^{2}(2 \alpha R)+ \\
& 8 \alpha^{4} R^{2} \omega I_{0}(\alpha R) I_{0}(2 \alpha R) I_{1}(2 \alpha R) \\
& +16 \alpha^{3} R \omega I_{0}(\alpha R) I_{1}^{2}(2 \alpha R) \\
& D_{2}=4\left(-\alpha^{2} R \omega+2 \alpha^{4} R^{3} \omega\right) I_{0}(\alpha R) I_{0}^{2}(2 \alpha R) \\
& -24 \alpha^{4} R^{3} \omega I_{0}(\alpha R) I_{1}^{2}(2 \alpha R)-16 \alpha^{3} R^{2} \omega I_{1}(\alpha R) I_{0}^{2}(2 \alpha R)+ \\
& 16 \alpha^{2} R \omega I_{1}(\alpha R) I_{0}(2 \alpha R) I_{1}(2 \alpha R) \\
& D_{3}=4 \alpha^{4} R^{2} \omega I_{0}^{2}(\alpha R) I_{1}(2 \alpha R) \\
& -8 \alpha^{3} R \omega I_{0}(\alpha R) I_{1}(\alpha R) I_{1}(2 \alpha R) \\
& +8 \alpha^{3} R \omega I_{1}^{2}(\alpha R) I_{0}(2 \alpha R) \\
& +4 \alpha^{4} R^{2} \omega I_{1}^{2}(\alpha R) I_{1}(2 \alpha R) \\
& -
\end{aligned}
$$


$D_{4}=-8 \alpha^{3} R^{2} \omega I_{0}^{2}(\alpha R) I_{1}(2 \alpha R)$

$-4 \alpha^{4} R^{3} \omega I_{0}^{2}(\alpha R) I_{0}(2 \alpha R)$

$+16 \alpha^{3} R^{2} \omega I_{0}(\alpha R) I_{1}(\alpha R) I_{0}(2 \alpha R)+$

$4\left(4 \alpha^{2} R \omega-\alpha^{4} R \omega\right) I_{0}(\alpha R) I_{1}(\alpha R) I_{1}(2 \alpha R)$

$-4\left(\alpha^{4} R \omega+2 \alpha^{2} R \omega\right) I_{1}^{2}(\alpha R) I_{0}(2 \alpha R)$

The following expressions for $v_{r}$ and $v_{z}$ can be written as

$v_{r}=\left[\alpha A_{1} r I_{0}(\alpha r)+\alpha B_{1} I_{1}(\alpha r)\right] \cos (\alpha z-\omega t)$

$+\left[2 \alpha A_{2} r I_{0}(2 \alpha r)+2 \alpha B_{2} I_{1}(2 \alpha r)\right] \cos 2(\alpha z-\omega t)$

$v_{z}=-\left[2 A_{1} r I_{0}(\alpha r)-\alpha A_{1} r^{2} I_{1}(\alpha r)+\alpha B_{1} r I_{0}(\alpha r)\right] \sin (\alpha z-\omega t)$

$-\left[2 A_{2} r I_{0}(2 \alpha r)-2 \alpha A_{2} r^{2} I_{1}(2 \alpha r)+2 \alpha B_{2} r I_{0}(2 \alpha r)\right] \sin 2(\alpha z-\omega t)$

\section{NUMERICAL RESULTS AND DISCUSSIONS}

In this section, numerical examples are presented. The test circular cylindrical shell and fluid flow have the following characteristics: $c=8.5 \mathrm{~m} / \mathrm{s}, \quad \omega=6 \pi \mathrm{rad} / \mathrm{s}$, $R=0.005 \mathrm{~m}$. The results are shown in Figs. (1-6).

Figs. (1-3) illustrate the fluid velocity $V_{z}$ from united Lagrangian-Eulerian method. Fig. (3) displays the variation of $V_{z}$ for several sets of values of the parameters $\omega, c, R, r$ and $z$ (see also Table 1). Moreover, Fig. (3) shows that fluid velocity $V_{z}$ increases with an increase in $\omega, R$ and $r$. However, it decreases with an increase in $c$.

Figs. (4-6) illustrate the fluid velocity $V_{r}$. Fig. (6) shows that the fluid velocity $V_{r}$ and $V_{z}$ have the same rules (see also Table 1). However, the positive and negative values of fluid velocity $V_{r}$ are asymmetrical.

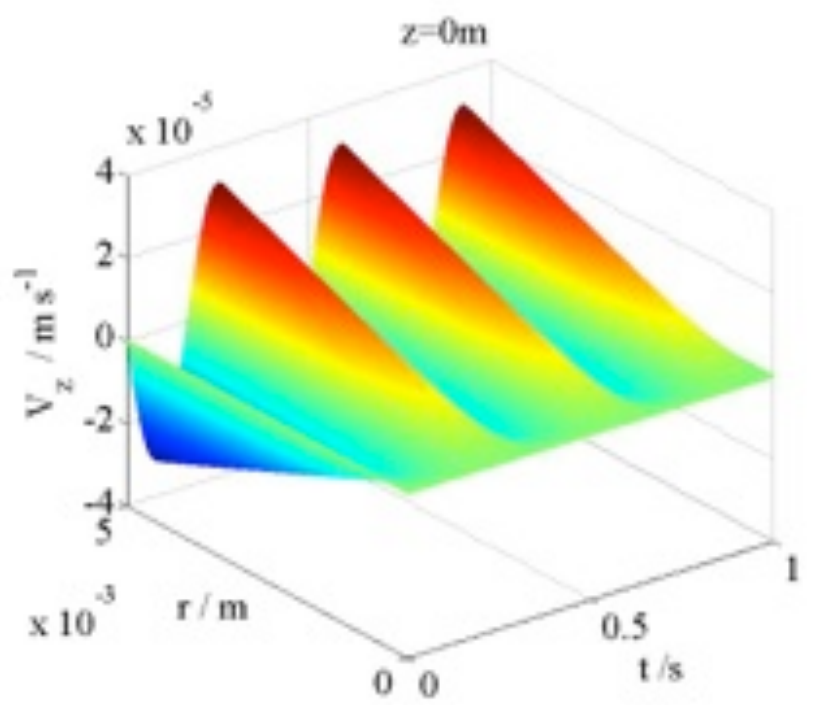

Fig. (1). Fluid velocity $V_{z}$ at $z=0 \mathrm{~m}$.

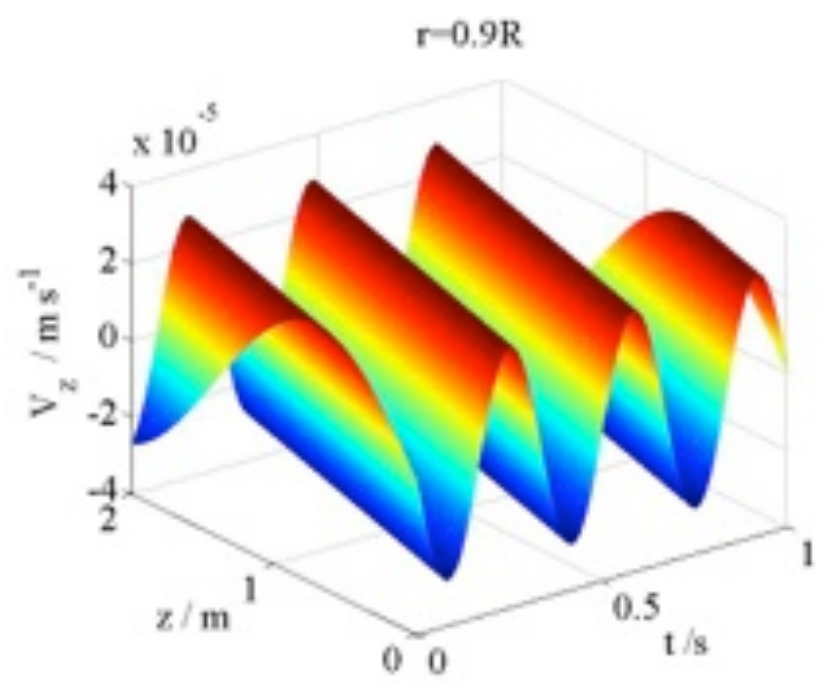

Fig. (2). Fluid velocity $V_{z}$ at $r=0.9 R$.

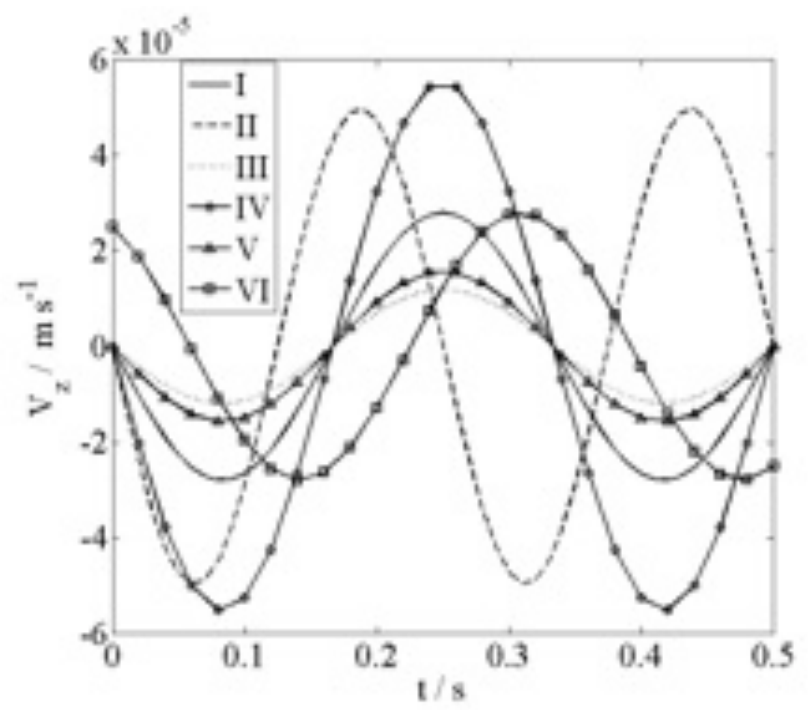

Fig. (3). Fluid velocity $V_{z}$.

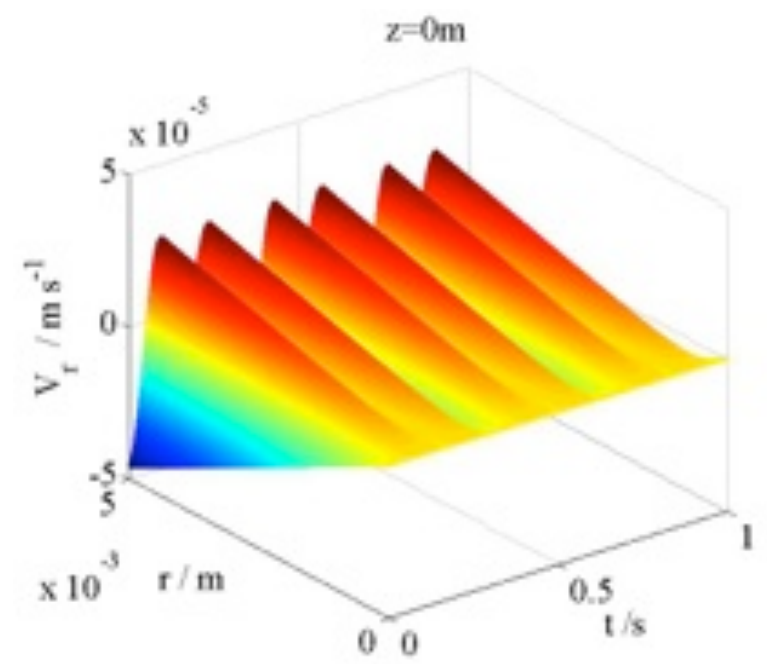

Fig. (4). Fluid velocity $V_{r}$ at $z=0 \mathrm{~m}$. 


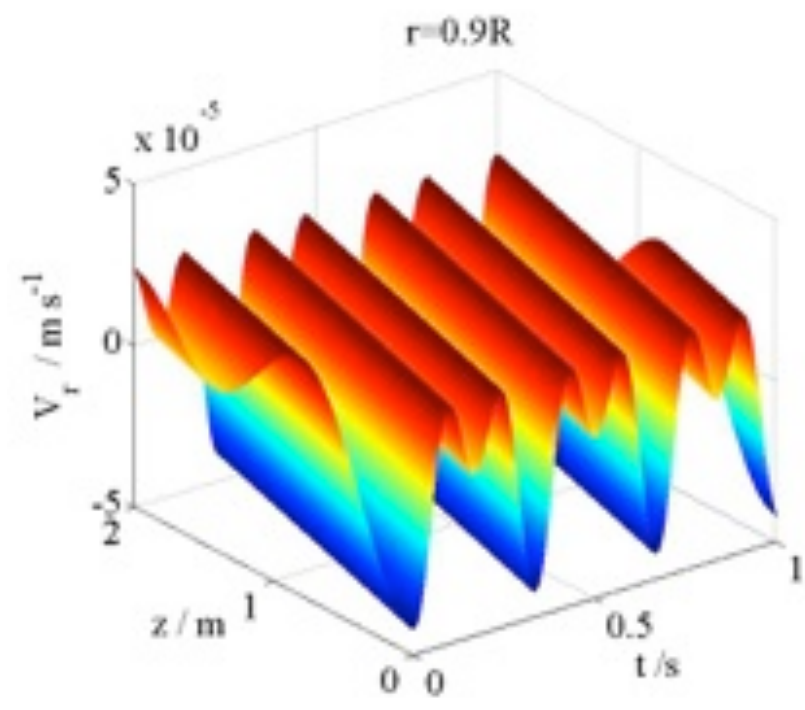

Fig. (5). Fluid Velocity $V_{r}$ at $r=0.9 R$.

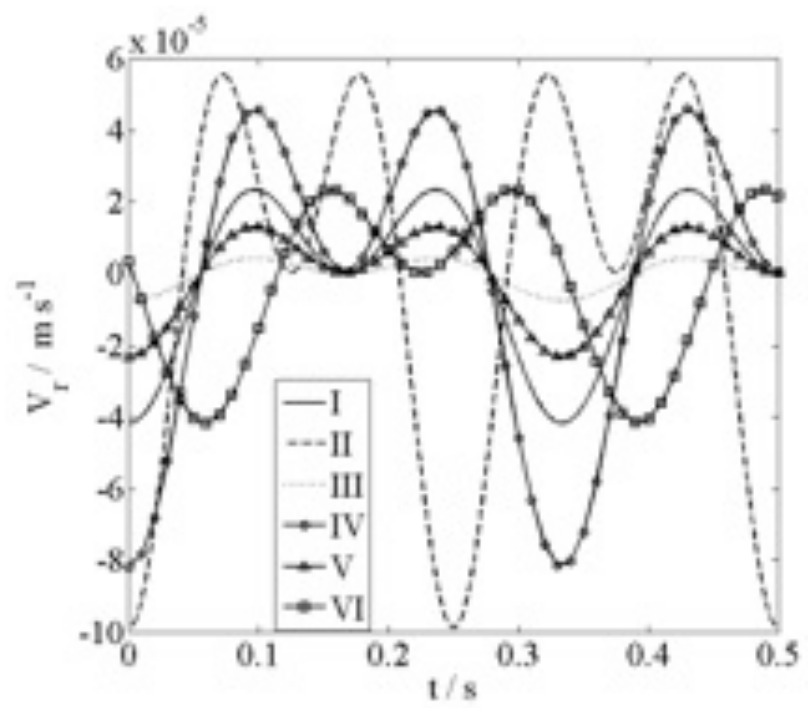

Fig. (6). Fluid Velocity $V_{r}$.

It is seen that a stagnation point exists where $V=0$ and the point $r=0$ in the circular cylindrical shell. A maximum velocity would exist near the surface of the circular cylindrical shell and the fluid. The fluid velocity is independent of the coefficient of viscosity. This result qualitatively agrees with the conclusions of the reference [9].
Table 1. Sets of parametric values for Figs. $(3,6)$.

\begin{tabular}{|c|c|c|c|c|c|}
\hline & $\begin{array}{c}\omega \\
(\mathbf{r a d} / \mathbf{s})\end{array}$ & $\boldsymbol{c}(\mathbf{m} / \mathbf{s})$ & $\boldsymbol{R}(\mathbf{m})$ & $\boldsymbol{r}(\mathbf{m})$ & $\boldsymbol{z}(\mathbf{m})$ \\
\hline \hline I & $6 \pi$ & 8.5 & 0.005 & 0.0045 & 0 \\
\hline II & $8 \pi$ & 8.5 & 0.005 & 0.0045 & 0 \\
\hline III & $6 \pi$ & 20 & 0.005 & 0.0045 & 0 \\
\hline IV & $6 \pi$ & 8.5 & 0.007 & 0.0045 & 0 \\
\hline V & $6 \pi$ & 8.5 & 0.005 & 0.0025 & 0 \\
\hline VI & $6 \pi$ & 8.5 & 0.005 & 0.0045 & 0.5 \\
\hline
\end{tabular}

\section{CONFLICT OF INTEREST}

The authors confirm that this article content has no conflict of interest.

\section{ACKNOWLEDGEMENTS}

This work is supported by National Natural Science Foundation of China (No. 11102181) and by Natural Science Foundation of Hebei Province (No. A2012203117).

\section{REFERENCES}

[1] M.Z. Chen, Viscous Fluid Dynamics, Higher Education Press, Beijing, 2001, pp. 111-120.

[2] Q. Gu, Method of Mathematical Physics, Science Press, Beijing, 2012, pp. 395-420.

[3] M.T. Heil, and J. Pedley, "Large axisymmetric deformation of a cylindrical shell conveying a viscous flow," Journal of Fluids and Structures, vol. 3, pp. 237-256, 1995.

[4] M.A. Iljgamov, The Introduction to Nonlinear Hydroelasticity, Moscow, Nauka, 1991, pp. 15-53.

[5] Y. Jin, and X. Yuan, "Numerical simulation of fluid-induced vibration in seals by fluid-structure coupling method," Journal of Engineering Thermophysics, vol. 3, pp. 395-398, 2003.

[6] V.N. Kuznetsov, "Interaction of an elastic cylindrical shell with a viscous fluid," Soviet Applied Mechanics, vol. 11, pp. 1228-1231, 1978.

[7] S. Lakovlev, "Submerged fluid-filled cylindrical shell subjected to a shock wave: Fluid-structure interaction effects," Journal of Fluids and Structures, vol. 1, pp. 117-142, 2007.

[8] J.Z. Ma, Y.R. Yang and J.Y. Zhang, "Investigation of dynamical characteristics of a core barrel in still water," Journal of Vibration Engineering, vol. 3, pp. 468-473, 2000.

[9] Y.L. Zhang, J.M. Reese and D.G. Gorman, "Finite element analysis of the vibratory characteristics of cylindrical shells conveying fluid," Computer Methods in Applied Mechanics and Engineering, vol. 45, pp. 5207-5231, 2002. 